\title{
Do advanced glycation end products contribute to saturated fat induced inflammation?
}

Obesity is associated with hypothalamic ${ }^{(1,2)}$ and peripheral ${ }^{(3)}$ inflammation. The hypothalamus is key in the regulation of appetite and energy homeostasis with dysfunction resulting in obesity. Dietary long chain saturated fatty acids (LCSFA) are causative in hypothalamic inflammation $^{(4,5)}$ but LCSFAs only appear effective in the presence of sugar via the formation of advanced glycation end products (AGEs) ${ }^{(6)}$. This raises a paradox as n-3 PUFAs, which are anti-inflammatory, are highly susceptible to AGE formation via lipid peroxidation. Thus, we investigated whether AGEs are pro inflammatory in the hypothalamic neuronal cell line, mHypoE-N42 (N42).

$\mathrm{N} 42$ cells were treated with $200 \mu \mathrm{M}$ palmitic (PA), oleic (OA), or eicosapentaenoic (EPA) for 6 hours ${ }^{(7)}$. N42 cells were also challenged with $100 \mu \mathrm{M}$ AGEs prepared as described by Castilho et al. ${ }^{(8)}$ for up to 24 hours. Gene expression of pro-inflammatory cytokines (IL6 and TNF $\alpha$ ) was measured by real-time quantitative RT-PCR using $B 2 m$ as a reference gene. Statistical analysis was performed by ANOVA followed by Students $t$-test.

PA upregulated $I L 6$ and $T N F \alpha$, whereas OA and EPA downregulated these genes (Fig. 1a and 1b). In contrast, AGE challenge downregulated IL6 and TNF $\alpha$ expression up to 6 hours followed by upregulation after 16 hours (Fig.1c and 1d). Gene expression and Western blotting showed the presence of receptor for AGE (RAGE) in cells and media confirming that cells are able to respond to AGEs.

(a)

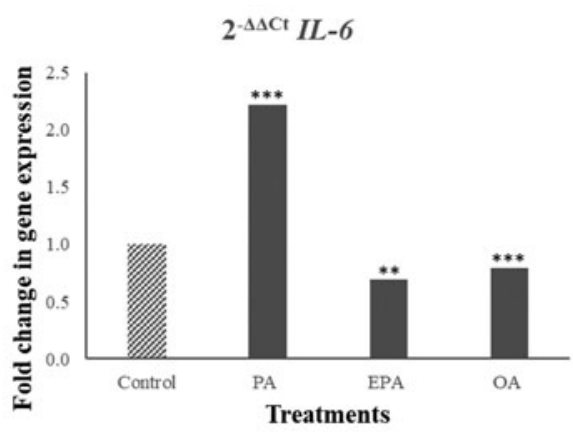

(b)

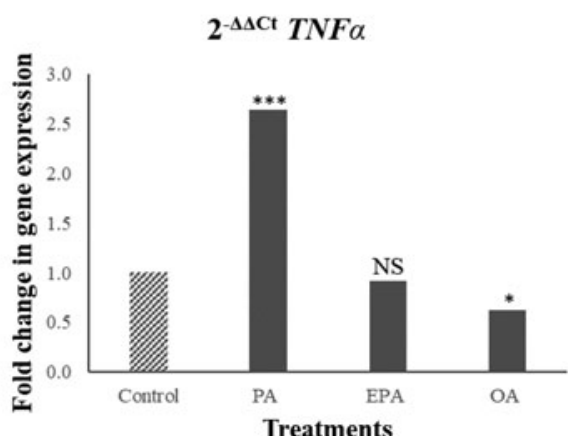

(c)

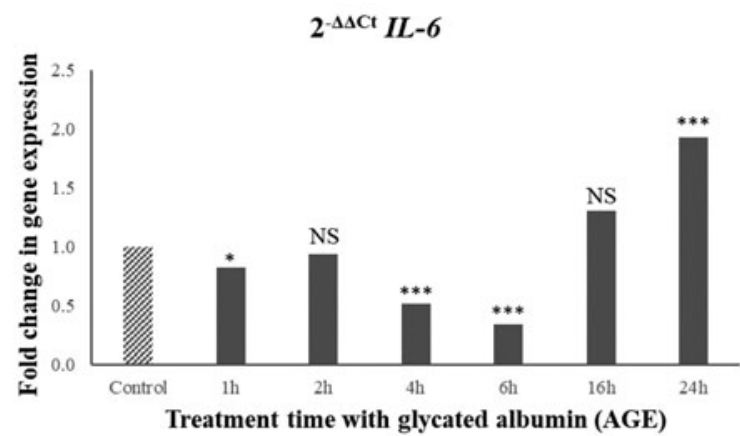

(d)

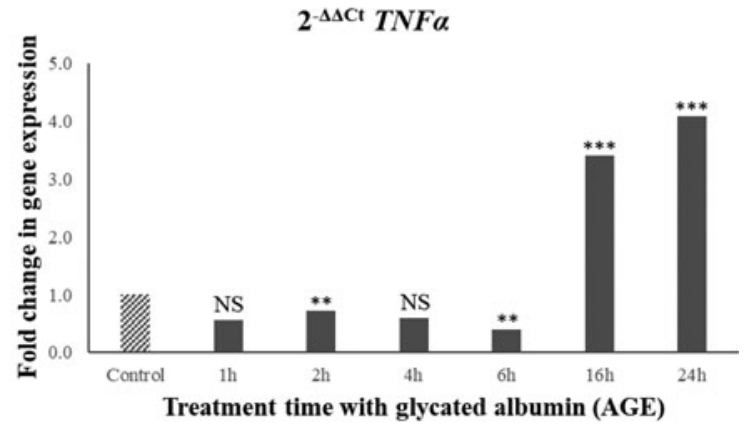

Fig. 1. Relative pro-inflammatory gene expression (IL6 and $T N F \alpha$ ) in the hypothalamic neurons after 6 hour fatty acid challenge (1a and $1 \mathrm{~b})$ or $1-24$ hours AGE challenge (1c and 1d). Values are ratios; stars indicate statistical significance of the differences between normalised threshold cycle number $\left(\Delta C_{t}\right)$ of control and treatments $(\mathrm{n}=4) .{ }^{*} \mathrm{p}<0.05 ; * * \mathrm{p}<0.01 ; * * \mathrm{*}<0.001$; NS non-significant.

Taken together, these results suggest that AGEs trigger pro-inflammatory responses in the hypothalamus on a different timescale compared to PA; and the pro-inflammatory effect of PA is not mediated via the formation of AGEs.

1. Arruda PA, Milanski M, Coope A et al. (2011) Endocrinology 152, 1314-1326.

2. Kreutzer C, Peters S, Schulte DM et al. (2017) Diabetes 66, 2407-2415.

3. McGuire TR, Brusnahan SK, Bilek LD et al. (2011) Obesity 19, 2130-2136.

4. Thaler JP, Yi C-X, Schur EA et al. (2012) J Clin Invest 122, 153-162.

5. De Souza CT, Araujo EP, Bordin S et al. (2005) Endocrinology 146, 4192-4199.

6. Gao Y, Bielohuby M, Fleming T et al. (2017) Mol Metab 6, 897-908.

7. Sergi D, Morris AC, Kahn DE et al. Nutr Neurosci. Published online: 21 July 2018. doi: 10.1080/1028415X.2018.1501533.

8. Castilho G, Okuda LS, Pinto RS et al. (2012) Int J Biochem Cell Biol 44, 1078-1086. 